Eur. J. Clin. Chem. Clin. Biochem.

Vol. 31, 1993, pp. 499-502

(C) 1993 Walter de Gruyter \& Co. Berlin - New York

\title{
Investigation of Total and Conjugated Bilirubin Determination during the Neonatal Period
}

\author{
By Jeanne Francoual ${ }^{1}$, Anne Myara ${ }^{2}$, Clarisse Benattar ${ }^{1}$, A. Ravaud ${ }^{3}$, Danièle Paumier ${ }^{2}$, J.-F. Magny ${ }^{4}$, \\ C. Messaoui ${ }^{3}$ and F. Trivin ${ }^{2}$ \\ 1 Service de Biochimie, Hôpital Antoine Beclere, Clamart, France \\ 2 Service de Biochimie, Hôpital Saint-Joseph, Paris, France \\ ${ }^{3}$ Service de Biochimie, Centre Hospitalier, Argenteuil, France \\ ${ }^{4}$ Service de Réanimation Néonatale, Hôpital Antoine Beclere, Clamart, France
}

(Received January 11/April 15, 1993)

Summary: During the neonatal period, total and conjugated bilirubin determinations are necessary to identify the origin of jaundice, to predict its evolution and to treat it. We discuss the results obtained in 108 neonates (less than 15 days old), undergoing phototherapy or not, using a colorimetric diazo reaction and dual wavelength reflectance with a Kodak Ektachem analyzer. Concerning total bilirubin determination, the methods correlate well ( $r>0.96$ ). Discrepancies are observed for conjugated or "direct" bilirubin, and high performance liquid chromatography was carried out in order to explain them. The chromatograms show 4 neonate samples with only classic mono- but no di-glucurono-conjugate fractions, whereas all the neonates present two unusual fractions (I and II) not seen in adults. A correlation was found between the amount of fraction II and the conjugated bilirubin determined by diazo reaction and between fraction I and the conjugated bilirubin obtained in the Kodak Ektachem assay. A better correlation between fraction I and conjugated bilirubin on Kodak was observed $(r=0.79$, vs $r=0.66)$ when the newborns were submitted to phototherapy. Moreover, fraction II and conjugated bilirubin measured by the diazo reaction on Hitachi 717 rose significantly.

In conclusion, total bilirubin is accurately determined during the neonatal period; for conjugated or "direct" bilirubin determination, our study points out significant differences. Further investigation will determine the nature of the fractions observed by liquid chromatography in neonatal sera, and the components actually determined by the automatized methods usually employed.

\section{Introduction}

Neonatal hyperbilirubinaemia is currently observed during the neonatal period ( $25-50 \%$ of all neonates), mostly due to low activity of the hepatic glucuronosyltransferase (EC 2.4.1.17) (1). Total and conjugated (or "direct") bilirubin determinations are necessary to identify the origin of the jaundice, to predict its evolution and to treat it. Visible light phototherapy is one of the effective treatments frequently given to these children, but it produces photochemical prod- ucts (2) that may interfere with the biochemical methods commonly used for bilirubin determination (3).

The aim of this study is to compare the data of total and conjugated bilirubin measured in neonates, undergoing phototherapy or not, using two commonly employed methods: colorimetric diazo reaction on a Hitachi 717, dual wavelength reflectance on a Kodak Ektachem 700. High performance liquid chromatography was then used for further investigation. 


\section{Materials and Methods}

\section{Patient samples}

Serum samples were from 108 neonates during the first 15 days of life (mean age: 4.7 days; range: $1-14$ days), submitted to the laboratory for a survey of the bilirubin course. The gestational ages of the infants ranged from 26 to 41 weeks (mean $33 \pm 4$ weeks). Among these neonates, 38 of them (35\%) were undergoing intermittent or continuous visible light phototherapy.

\section{Bilirubin analysis procedures}

For the colorimetric method, bilirubin is coupled with diazotized sulphanilic acid, in the presence (total bilirubin determination) or in the absence (direct bilirubin determination) of caffeine, to yield an azodye (Boehringer Mannheim $\mathrm{GmbH}$, Germany) (4). This was done on a Hitachi 717 automatic analyzer (Hitachi, Ltd, Tokyo, Japan). For calibration, a commercially available solution containing direct and unconjugated bilirubin was used (Bilitrol Biomerieux, France).

For the dual wavelength reflectance method, unconjugated and conjugated bilirubin concentrations were determined using the dry slide $\mathrm{Bu}-\mathrm{Bc}$ on a Kodak Ektachem 700 analyzer (Eastman Kodak Company, Rochester-Ny, USA), according to the manufacturer's recommendations for samples from newborns less than 15 days old. Total bilirubin was calculated by adding unconjugated and conjugated bilirubin (5).

The high performance liquid chromatography procedure used derives from the method described by Lauff et al. (6). After precipitation of the high molecular mass proteins by $\mathrm{Na}_{2} \mathrm{SO}_{4}$, different fractions of bilirubin are separated on a Lichrosorb RP $8(125 \times 4 \mathrm{~mm})$ Merck column, using an HP 1090 apparatus. A step gradient solvent is used for elution. Retention times of $4.86,6.36,7.63$ and 11.08 minutes are, respectively, characteristic for delta, di-, mono-glucurono-conjugated and unconjugated bilirubin. Each fraction is quantified by a diode array detector at $450 \mathrm{~nm}$ by comparison with different bilirubin standard solutions.

The bilirubin concentrations are given in $\mu \mathrm{mol} / \mathrm{l}$. During the tests all serum samples are protected against light.

\section{Statistics}

The data obtained with the different methods were compared, using regression analysis. The results of bilirubin determination for newborns with or without phototherapy were compared using variance analysis (ANOVA).

\section{Results}

Results of a precision study of bilirubin determination by the different methods are summarized in table 1.

Tab. 1. Precision of bilirubin determination by the different methods.

\begin{tabular}{lll}
\hline & $\begin{array}{l}\text { Total } \\
\text { bilirubin }\end{array}$ & $\begin{array}{l}\text { "Direct" } \\
\text { bilirubin }\end{array}$ \\
& CV (\%) & CV (\%) \\
\hline Diazo reaction & 2.5 & 5.1 \\
Dual wavelength reflectance & 3.5 & 4.3 \\
HPLC & 1.7 & 2.0 \\
\hline
\end{tabular}

The mean total bilirubin, determined by means of the two automated analyzers (Hitachi 717 and Kodak Ektachem 700) were, respectively, $160 \pm 61 \mu \mathrm{mol} / 1$ and $156 \pm 58 \mu \mathrm{mol} / \mathrm{l}$, which correlated well (fig. 1).

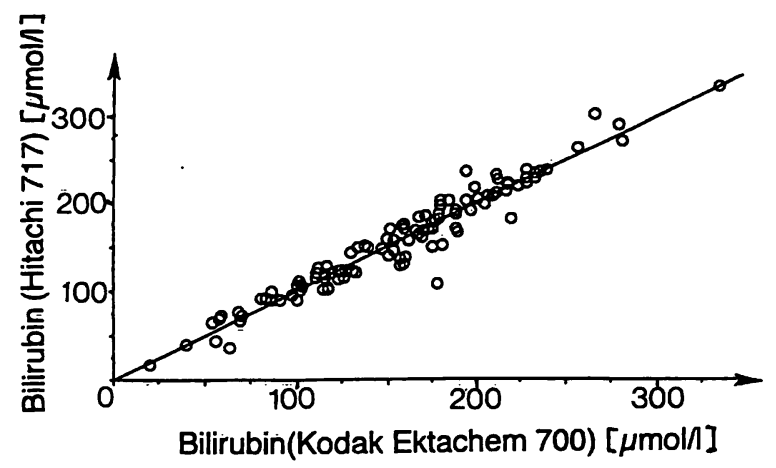

Fig. 1. Correlation study for total bilirubin. $\mathrm{y}=1.009 \mathrm{x}-0.789, \mathrm{r}=0.97$.

For conjugated or "direct" bilirubin, data are shown in table 1 . The concentrations of conjugated or "direct" bilirubin ranged from 0 to $40 \mu \mathrm{mol} / 1$, and no correlation was found between the results obtained by the two automated analyzers (fig. 2). In order to explain these discrepancies, we performed reversephase high performance liquid chromatography. The chromatograms show that delta bilirubin was always less than $1 \%$ of the total bilirubin, that di-glucuronoconjugated fractions were not observed and that the usual mono-glucurono-conjugated fractions were present only in 4 neonates $(<4 \mu \mathrm{mol} / \mathrm{l})$. Two of the neonates were 4 days old, the others 5 and 11 days old.

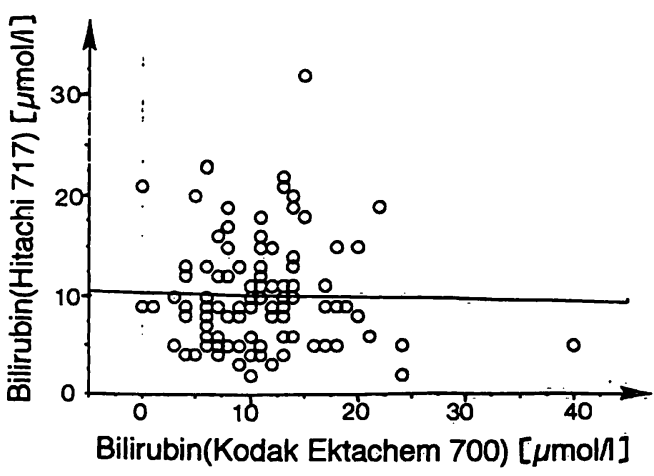

Fig. 2. Correlation study for "direct" bilirubin. $y=-0.0231 x+10.429$

We found a particular profile for all the neonatal serum samples (fig. 3). Two fractions with retention times of 5.94 and 8.05 minutes were observed, which we called neonate fraction I and II. Their spectra are compatible with those of bilirubin fractions (absorbance between 420 and $460 \mathrm{~nm}$ ). The comparison, by means of regression analysis, of these fractions with 


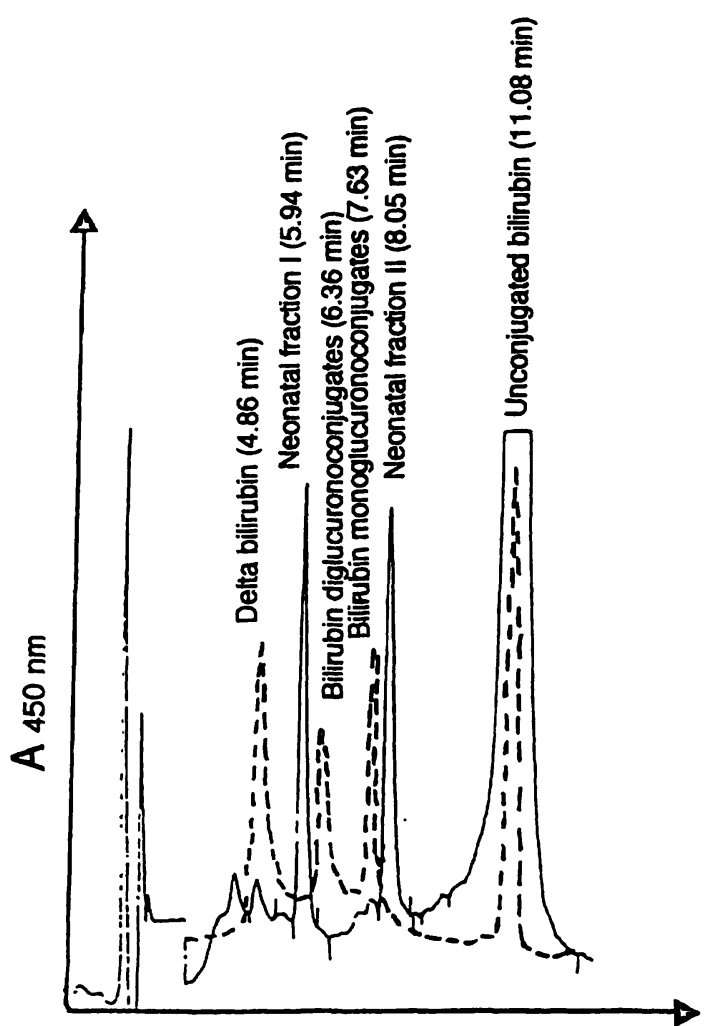

Retention time

Fig. 3. HPLC bilirubin profiles obtained with adult (--) and neonate $(-)$ sera.
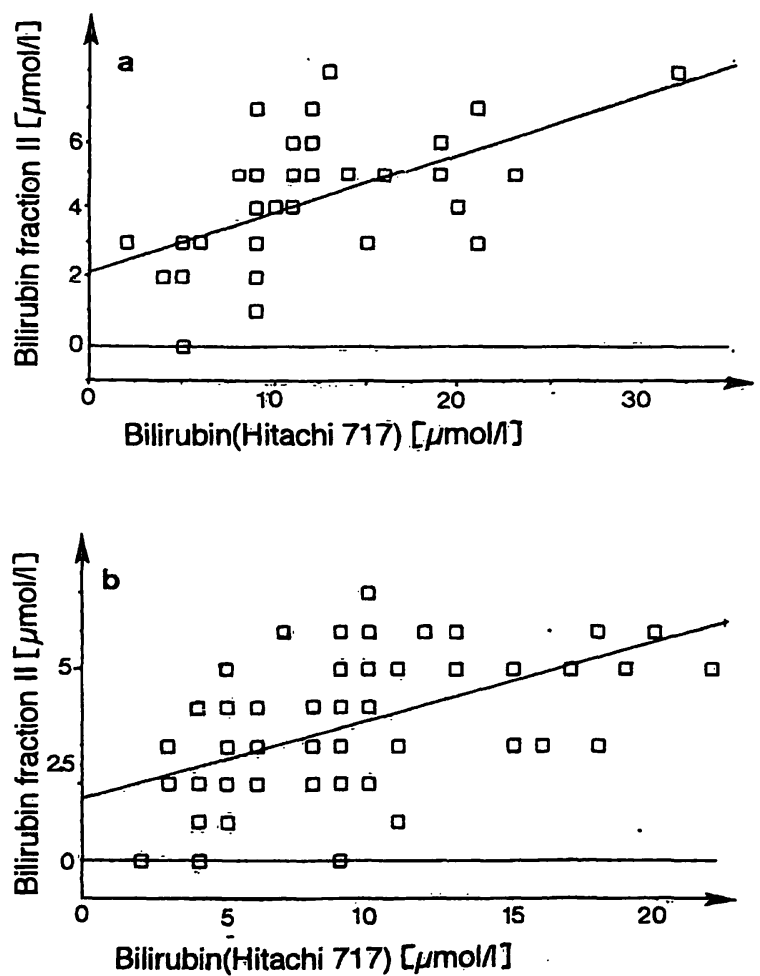

Fig. 4. Correlation between fraction II and direct bilirubin determination by diazo reaction.

a: with phototherapy $y=0.175 x+2.106, r=0.56$

b: without phototherapy, $y=0.206 x+1.592, r=0.52$ the conjugated or "direct" bilirubin obtained by the two other assays previously described, shows that each method separately correlates only with one of the two neonate fractions: fraction II with diazo reaction (fig. 4) and fraction I with the dual-wavelength reflectance assay. This is particularly true in the latter case when the newborns are undergoing phototherapy (fig. 5). Moreover, when the newborns received this treatment, fraction II and the rate of conjugated bilirubin determined by diazo reaction rose significantly $(\mathrm{p}<0.05)$ (tab. 2).

Finally, no correlation was found between the presence of conjugated bilirubin (whatever the methodology) and the gestational age of the newborns.

\section{Discussion}

Total serum bilirubin is accurately determined during the neonatal period (less than 15 days of life) by the two automated assays: dual wavelength reflectance and the diazo reaction method, as previously described (7).
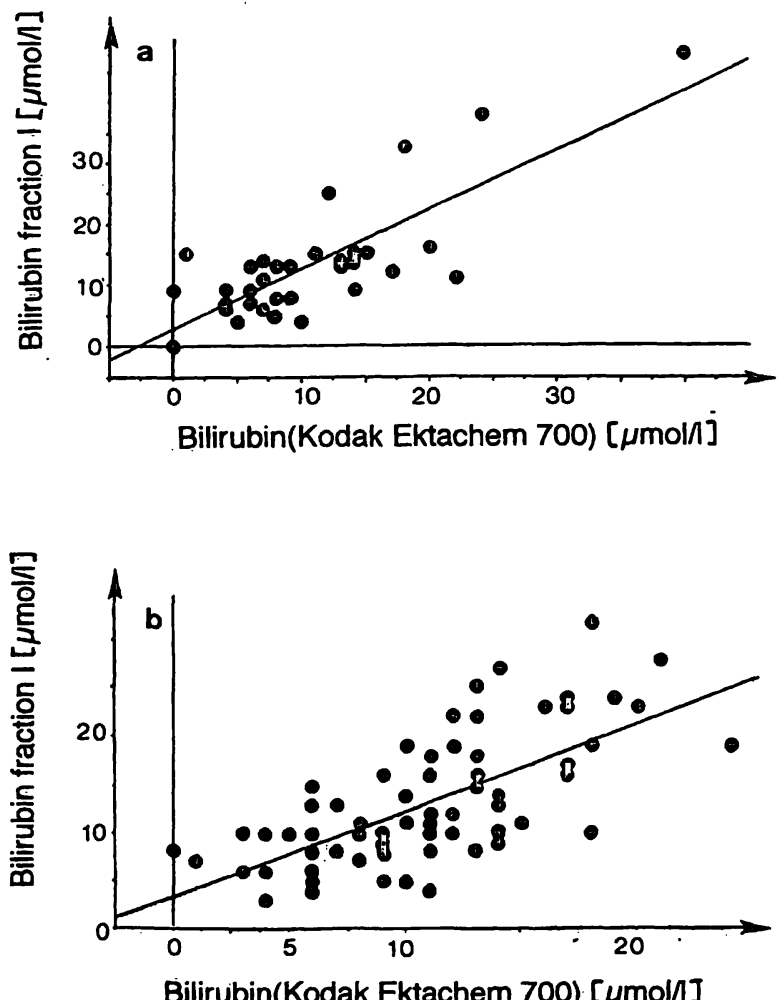

Fig. 5. Correlation between fraction I and direct bilirubin determination on Kodak analyzer.

a: with phototherapy $y=0.975 x+2.788, r=0.79$

b: without phototherapy $y=0.907 x+3.226, r=0.66$ 
Tab. 2. Conjugated or "direct" bilirubin ( $\mu \mathrm{mol} / 1)$ data obtained from 108 neonate samples.

\begin{tabular}{|c|c|c|c|c|c|}
\hline & $\cdot$ & Hitachi 717 & Kodak & $\begin{array}{l}\text { HPLC } \\
\text { Fraction I }\end{array}$ & $\begin{array}{l}\text { HPLC } \\
\text { Fraction II }\end{array}$ \\
\hline $\begin{array}{l}\text { Neonates without phototherapy } \\
n=73\end{array}$ & $\begin{array}{l}\text { Mean } \pm S D \\
\text { Range }\end{array}$ & $\begin{array}{l}9.4 \pm 4.3 \\
2 \quad-22\end{array}$ & $\begin{array}{c}10.6 \pm 4.8 \\
0=24\end{array}$ & $\begin{array}{c}12.4 \pm 6.4 \\
3-32\end{array}$ & $\begin{array}{ll}3.5 & \pm 1.7 \\
0 & -7\end{array}$ \\
\hline $\begin{array}{l}\text { Neonates undergoing phototherapy } \\
n=35\end{array}$ & $\begin{array}{l}\text { Mean } \pm S D \\
\text { Range }\end{array}$ & $\begin{array}{c}14.0^{*} \\
2\end{array}$ & $\begin{array}{c}10.7 \pm 7.9 \\
0 \leq 40\end{array}$ & $\begin{array}{c}12.5 \pm 9.1 \\
0=48\end{array}$ & $\begin{array}{l}4.5^{*} \pm 2.0 \\
0=8\end{array}$ \\
\hline
\end{tabular}

* Significant difference $(p<0.05)$ between neonates with and without phototherapy

With regard to determination of the fraction called conjugated or "direct" bilirubin, some questions still remain and the results are not so clear-cut. With HPLC the chromatographic profile differs depending on whether the samples are obtained from neonates or from adults. The di-glucurono-conjugated fraction in particular is never observed and the mono-glucurono-conjugated fraction is seen only in a few cases $(n=4)$, at aiconcentration always less than $4 \mu \mathrm{mol} / \mathrm{l}$. Rosenthal et al. (8) described conjugated fractions found during the neonatal period, when using a different high performance liquid chromatographic procedure and a serum sample far larger than we were able to apply.

In order to explain the origin of the two particular neonatal fractions (I and II), various hypotheses can be suggested. The hyperbilirubinaemia of neonates might be a consequence of an impaired glucuronidation; other sugar conjugates of bilirubin could be produced (7). These polar compounds might behave like glucuronated bilirubin with the diazo reaction or dual wavelength reflectance. Another alternative is the appearance of photo-derivatives or haemoglobin components likely to be present in haemolyzed samples, interfering with conjugated bilirubin determination. During phototherapy, the analysis of bile

\section{References}

1. Cornelius, C. E. (1988) Bilirubin metabolism in fetus and neonate. In: Non-Human Primates in Perinatal Research (Brans, Y. W. \& Kuehl, T. J., eds.) pp. 393-410, Wiley and Sons.

2. McDonagh, A. F. \& Lightner, D. A. (1985) "Like a shrivelled blood orange" - Bilirubin, jaundice, and phototherapy. Pediatrics $75,443-455$.

3. Ihara, H., Nakamura, H., Aoki, Y., Aoki, T. \& Yoshida, M. (1992) In vitro effects of light on serum bilirubin subfractions measured by high performance liquid chromatography: Comparison with four routine methods. Clin. Chem. 38, 2124-2129.

4. Jendrassik, L. \& Grof, P. (1938) Vereinfachte photometrische Methoden zur Bestimmung des Blutbilirubins. Biochem. Z. 297, 81-89.

5. Wu, T. W., Dappen, G. M., Spayd, R. W., Sundberg, M. W. \& Powers, D. M. (1984) The Kodak Ektachem clinical chemistry slide for simultaneous determination of unconjugated and sugar-conjugated bilirubin. Clin. Chem. 30, 1304-1309.

6. Lauff, J. J., Kasper, M. E. \& Ambrose, R. T. (1981) Separation of bilirubin species in serum and bile by high shows that lumirubin is the major bilirubin isomer excreted (9), but configurational isomers may also be present in the circulation. These different components, specific for the neonatal period, may interfere with the determination of conjugated or "direct" bilirubin by the usual methods and further investigation is necessary to determine their real nature.

Moreover, the diazo reaction determines the part of bilirubin that reacts "directly" with diazotized sulphanilic acid, whereas the reflectance method evaluates the absorbance of conjugated bilirubin derivates $(5,7)$.

In conclusion, a discrepancy is observed between two methods commonly used (i. e., diazo reaction and dual wavelength reflectance) for the determination of "conjugated" bilirubin in neonatal samples. When these samples are analyzed by liquid chromatography, the usual fractions are rarely observed, whereas two different fractions are present (I and II) that require further investigation to determine their real nature and to assess their role in the above-mentioned discrepancy. Finally, these results show that the determination of "direct bilirubin" during the neonatal period involves still unresolved problems as regards the study of premature newborns (mean $=33$ weeks) or term ones (10).

performance reversed-phase liquid chromatography. J. Chromatogr. 226, 391-402.

7. Doumas, B. T. \& Wu, T. W. (1991) The measurement of bilirubin fractions in serum. CRC in Clin. Lab. Sci. 28 , 415-445.

8. Rosenthal, P., Blanckaert, N., Kabra, P. M. \& Thaler, M. M. (1986) Formation of bilirubin conjugates in human newborns. Pediatr. Res. 20, 947-950.

9. Ennever, J. F., Costarino, A. T., Polin, R. A. \& Speck, W. T. (1987) Rapid clearance of a structural isomer of bilirubin during phototherapy. J. Clin. Invest. 79, 1674-1678.

10. Newman, T. B., Hope, S. \& Stevenson, D. K. (1991) Direct bilirubin measurements in jaundiced term newborns - A reevaluation. Amer. J. Dis. Child. 145, 1305-1309.

Anne Myara
Service de Biochimie
Hôpital Saint-Joseph
7, rue Pierre Larousse
F-75674 Paris
France

Eur. J. Clin. Chem. Clin. Biochem. / Vol. 31, 1993 / No. 8 